\title{
The impact of the last ten minutes of surgery on hemorrhagic complications after laparoscopic sleeve gastrectomy. Case-control study
}

\author{
Michał R. Janik, Mateusz Czado, Konrad Kosiński, Szymon Grochans, Maciej Walędziak, Piotr Kowalewski, \\ Andrzej Kwiatkowski \\ Department of General, Oncologic, Metabolic and Thoracic Surgery, Military Institute of Medicine, Warsaw, Poland
}

Videosurgery Miniinv 2021; 16 (3): 566-570

DOI: https://doi.org/10.5114/wiitm.2021.104012

\begin{abstract}
Introduction: Hemorrhagic complications after laparoscopic sleeve gastrectomy (LSG) are among the most common adverse events. The last 10 min of LSG are essential in terms of hemostasis.

Aim: To assess the blood pressure profile in the last 10 min of LSG in patients who experienced hemorrhagic complications after laparoscopic sleeve gastrectomy.

Material and methods: We performed a retrospective case-control study. The medical records of 867 patients who underwent primary LSG were analyzed. Cases were defined as patients who required surgical revision due to hemorrhagic complications within $72 \mathrm{~h}$. Controls were matched (1:1) with cases by age, body mass index, gender, staple line reinforcement, comorbidities and surgeon's experience. Comparison of the last three intraoperative blood pressure measurements at the end of surgery was made.

Results: The bleeding rate was 3.0\%. A total of 24 subjects (12 matched pairs) were included in the study. Cases had statistically significant increased mean arterial blood pressure $(\mathrm{mm} \mathrm{Hg}) 5 \mathrm{~min}$ before the end of surgery $(87.8 \pm 11.9 \mathrm{vs}$. $79.4 \pm 8.8 \mathrm{~mm} \mathrm{Hg}, p=0.049)$ and at the end of surgery $(89.2 \pm 11.7 \mathrm{vs} .77 .5 \pm 11.8 \mathrm{~mm} \mathrm{Hg}, p=0.011)$. Higher diastolic blood pressure measurements were observed 5 min before the end $(72.1 \pm 10.7 \mathrm{vs} .62 .8 \pm 8.1 \mathrm{~mm} \mathrm{Hg}, p=0.023)$ and at the end of surgery (74.2 $\pm 10.0 \mathrm{vs.} 60.8 \pm 11.2 \mathrm{~mm} \mathrm{Hg}, p=0.004)$.

Conclusions: Compared with closely matched control subjects, patients with HC after LSG have increased mean arterial pressure in the last 5 min of surgery. This phenomenon has not been reported in the literature before.
\end{abstract}

Key words: laparoscopic sleeve gastrectomy, complications, bariatric surgery, bleeding.

\section{Introduction}

Laparoscopic sleeve gastrectomy (LSG) is the most popular bariatric procedure $[1,2]$. It gained popularity amongst surgeons due to simplicity. The long term weight loss and metabolic outcomes of LSG are generally good [3-5]. Adverse events include gastric leak, stenosis and postoperative bleeding. The bleeding rate after LSG varies from $1 \%$ to $5 \%$ [6].
Although hemorrhagic complications are among the most common adverse events, the issue is not explored enough. Our previous study indicated that hypertension is a significant risk factor for postoperative bleeding in patients after LSG [7]. This finding leads us to further studies on bleeding complications and blood pressure measurements. The analysis of postoperative blood pressure profile showed opposite results to the popular belief that patients 
with hypertension in the postoperative period are more likely to have bleeding complications [8]. This case-control study of intraoperative blood pressure was undertaken to investigate the association between blood pressure at the end of the surgical procedure and postoperative bleeding.

\section{Aim}

The study aimed to assess the blood pressure profile in the last 10 min of LSG in patients who experienced hemorrhagic complications after laparoscopic sleeve gastrectomy.

\section{Material and methods}

\section{Study design}

We performed a retrospective case-control study. The medical records of 867 patients who underwent primary laparoscopic sleeve gastrectomy were analyzed. Patients underwent surgery between January 2013 and December 2017 in a single bariatric unit. Part of this cohort was used in our previous publications [9]. We decided to focus on the last $10 \mathrm{~min}$ of surgery - defined as $10 \mathrm{~min}$ before the end of anaesthesia. It is the time when a surgeon assesses a surgical field and performs homeostasis before the surgery ends. The period also includes the hemodynamic changes resulting from gas exsufflation and the changing of the patients' position.

\section{Participants - subjects}

Cases were defined as patients who required surgical revision due to hemorrhagic complications within 72 h. Hemorrhagic complications included bleeding from different sources, including the staple line, omentum, short gastric vessels and abdominal wall as well as the presence of a large hematoma [10]. We identified 26 cases of patients with hemorrhagic complications. Patients who were taking anticoagulation drugs were excluded.

\section{Participants - normal controls}

Controls patients were taken retrospectively from the rest of the subjects who underwent LSG between January 2013 and December 2017 and did not experience any of the following complications: hemorrhagic complications, gastric leakage or sleeve stenosis. All controls identified as potential matched controls for the cases.

\section{Matching}

Controls were matched $(1: 1)$ with cases by age ( \pm 3 year), BMI $\left( \pm 4 \mathrm{~kg} / \mathrm{m}^{2}\right)$ gender (female versus male), staple line reinforcement (running suture versus hemostatic clips), comorbidities (diabetes, hypertension and obstructive sleep apnea) and surgeon's experience (> 50 or < 50 LSG procedures per year). A control subject was selected for each patient with $\mathrm{HC}$ using the algorithm described by Kawabata (1: 1 matching method) [11]. Cases for whom we could not identify suitable matching control subjects were excluded from the study.

\section{Surgical technique and measurements}

Surgical technique and postoperative management were described in our previous publications [9]. In all cases, we used $15 \mathrm{~mm} \mathrm{Hg}$ intrabdominal pressure for pneumoperitoneum. The automatic blood pressure measurements were recorded within every $5 \mathrm{~min}$. We compared the last three blood pressure measurements $-\left(1^{\text {st }}\right)$ ten and $\left(2^{\text {nd }}\right) 5$ min before the end of surgery and at the end of the surgery $\left(3^{\text {rd }}\right)$. The fist measurement corresponds with surgical field inspection for bleeding. The second and third measurements correspond with the hemodynamic changes resulting from exsufflation and change of reverse Trendelenburg to the supine position.

\section{Bias}

Observers were blind to the exposure, and the data were collected retrospectively from medical records by a single investigator. The risk of information bias is low. To minimize selection bias, we analyzed a large number of cases of primary LSG. Regarding the reports, LSG constitutes about 800 bariatric procedures per year in Poland [2]. Thus, our data are representative. All patients underwent the same procedure according to the standard surgical technique of LSG described before, which eliminate referral bias. The study size was dependent on the number of patients with $\mathrm{HC}$ who were matched with controls.

\section{Statistical analysis}

The analysis was performed using SAS software, University Edition (SAS Institute Inc., Cary, NC, USA). Continuous outcomes were analyzed using the paired $t$-test, or Wilcoxon signed ranks test. Dichot- 
Table I. Demographic characteristics of analyzed patients $(n=867)$

\begin{tabular}{|lc|}
\hline Characteristics & Mean (SD) or \% \\
\hline Age [years] & $40.7(11.8)$ \\
\hline BMI [kg/m²] & $46.2(7.4)$ \\
\hline Gender (female) & $487(56.2 \%)$ \\
\hline Hypertension & $413(50.8 \%)$ \\
\hline Diabetes & $161(19.2 \%)$ \\
\hline Dyslipidemia & $87(10.4 \%)$ \\
\hline OSA & $49(5.9 \%)$ \\
\hline LOS [days] & $3.7(1.6)$ \\
\hline Mortality & $3(0.3)$ \\
\hline Leak & $26(3.0 \%)^{*}$ \\
\hline HC (from 2013 to 2017): & $3 / 146(2.0 \%)$ \\
\hline 2013 & $14 / 188(6.9 \%)$ \\
\hline 2014 & $4 / 175(2.2 \%)$ \\
\hline 2015 & $3 / 198(1.5 \%)$ \\
\hline 2016 & $2 / 137(1.4 \%)$ \\
\hline 2017 & $35)$ \\
\hline
\end{tabular}

*The $p$-value for observed differences of HC incidence from 2013 to 2017 was significant (0.019). omous outcomes were analyzed using McNemar's test. The analysis of matched (dependent) data is different from unmatched (independent) data and is described in detail by Breslow and Day [12].

\section{Results}

The cumulative bleeding rate was 3.0\%. However, we observed changes in this rate between years. The highest percentage (6.9\%) was noted in 2014. It was associated with the beginning of the learning curve for new surgeons, who were introduced to the bariatric practice that year. A total of 24 subjects (12 matched pairs) were included in the study. Table I shows the descriptive characteristics of the patients. The groups were closely matched as expected (Table II). In 9 cases, the source of bleeding was identified. In 6 cases the bleeding was from the staple line, in 2 cases from short gastric vessels and in 1 case from splenic injury (Table III). Patients who experienced hemorrhagic complications after LSG had statistically significantly increased mean arterial blood pressure $(\mathrm{mm} \mathrm{Hg}) 5 \mathrm{~min}$ before the end of surgery $(87.7 \pm 11.9$ vs. $79.4 \pm 8.8 \mathrm{~mm} \mathrm{Hg}, p=0.049)$ and the

Table II. Demographic characteristics of matched patients

\begin{tabular}{|c|c|c|c|}
\hline Characteristics & Patients with HC $(n=12)$ & Controls subjects $(n=12)$ & $P$-value \\
\hline Age [years] & $42.0 \pm 11.2$ & $42.5 \pm 11.3$ & 0.437 \\
\hline $\mathrm{BMI}\left[\mathrm{kg} / \mathrm{m}^{2}\right]$ & $43.5 \pm 5.9$ & $43.6 \pm 4.8$ & 0.970 \\
\hline \multicolumn{4}{|l|}{ Gender: } \\
\hline Female & $6(50 \%)$ & $6(50 \%)$ & \multirow[t]{2}{*}{-} \\
\hline Male & $6(50 \%)$ & $6(50 \%)$ & \\
\hline \multicolumn{4}{|l|}{ Comorbidities: } \\
\hline Hypertension & $6(50 \%)$ & $6(50 \%)$ & - \\
\hline Diabetes & $2(17 \%)$ & $2(17 \%)$ & - \\
\hline Dyslipidemia & $1(8 \%)$ & $1(8 \%)$ & - \\
\hline OSA & $0(0 \%)$ & $0(0 \%)$ & \\
\hline \multicolumn{4}{|c|}{ Staple line reinforcement: } \\
\hline Hemostatic clips & $8(67 \%)$ & $8(67) \%$ & \multirow[t]{2}{*}{-} \\
\hline Running suture & $4(33 \%)$ & $4(33 \%)$ & \\
\hline \multicolumn{4}{|c|}{ Surgeon's experience: } \\
\hline Low & $5(42 \%)$ & $5(42 \%)$ & \multirow[t]{2}{*}{-} \\
\hline High & $7(58 \%)$ & $7(58 \%)$ & \\
\hline
\end{tabular}


end of surgery $(89.2 \pm 11.7$ vs. $77.5 \pm 11.8 \mathrm{~mm} \mathrm{Hg}$ $p=0.011$ ). The detailed analysis revealed significantly higher diastolic blood pressure measurements 5 min before the end $(72.1 \pm 10.7$ vs. $62.8 \pm 8.1 \mathrm{~mm}$ $\mathrm{Hg}, p=0.023)$ and at the end of surgery $(74.2 \pm 10.0$ vs. $60.8 \pm 11.2 \mathrm{~mm} \mathrm{Hg}, p=0.004$ ) (Table IV).

We observed a clear increasing tendency in mean arterial pressure, systolic and diastolic blood pressure in cases over the last 5 min of surgery, while in controls this tendency was the opposite (Figure 1).

\section{Discussion}

This study reports the blood pressure profile in the last 10 min of LSG in patients who experienced hemorrhagic complications, compared with closely matched controls. Data were extracted out of the retrospectively collected database of patients who underwent LSG between January 2013 and May 2017 in a single bariatric unit.

We hypothesized that low blood pressure at the end of surgery might lead to inadequate assessment of bleeding, which should be stopped before the procedure is ended. However, in the first measurement, there was no significant difference. It means that during the bleeding assessment, the blood pressure was comparable. Our hypothesis failed. Surprisingly, we observed an increasing tendency in mean arterial pressure, and diastolic blood pressure in cases over the last 5 min of surgery, while in controls this tendency was opposite. Even though the results were statistically significant, the observed changes were not clinically meaningful. The differences were too small to be useful in daily practice. In our opinion,
Table III. Source of bleeding $(n=12)$

\begin{tabular}{|lcc|}
\hline Source of bleeding & $N$ & $\%$ \\
\hline Unknow & 3 & 25.00 \\
\hline Staple line & 6 & 50.00 \\
\hline Short gastric vessels/Omentum & 2 & 16.66 \\
\hline Spleen & 1 & 8.33 \\
\hline
\end{tabular}

the phenomenon indicates that the two groups differ in the way they react to gas exsufflation. The observation may indicate the next direction for future studies on the hemodynamic changes during exsufflation.

Altered physiology has been demonstrated during laparoscopy in obese patients. The physiological changes are a result of patient position, absorption of $\mathrm{CO}_{2}$, and increased intra-abdominal pressure due to pneumoperitoneum. Pneumoperitoneum affects intraoperative hemodynamics and cardiac function in morbidly obese patients. Heart rate, mean arterial pressure and central venous pressure increased [13]. At the end of laparoscopic bariatric surgery, there are two main events which provoke hemodynamic changes - exsufflation and change of reverse Trendelenburg to the supine position. A study group investigating the effect of laparoscopy in cholecystectomy on hemodynamics observed that the fall in mean arterial pressure after exsufflation could result from the reversal of the impact of $\mathrm{CO}_{2}$ pneumoperitoneum [14]. There were no similar studies on bariatric procedures.

Interestingly, in our study patients who experienced hemorrhagic complications after LSG tended

Table IV. Blood pressure measurements of last 10 min of surgery

\begin{tabular}{|c|c|c|c|}
\hline \multirow[t]{2}{*}{ Variable } & \multicolumn{3}{|c|}{ Last 15 min of surgery } \\
\hline & $10 \mathrm{~min}$ before the end & 5 min before the end & 15 min before the end \\
\hline \multicolumn{4}{|c|}{ Mean arterial pressure [mm Hg]: } \\
\hline Cases & $84.4(7.7)$ & $87.7(11.9)^{\star}$ & $89.2(11.7)^{\star \star}$ \\
\hline Control & $83.6(9.6)$ & $79.4(8.8)^{\star}$ & $77.5(11.8)^{\star \star}$ \\
\hline \multicolumn{4}{|c|}{ Systolic blood pressure [mm Hg]: } \\
\hline Cases & $117.5(13.4)$ & $119.2(19.5)$ & $119.2(17.3)$ \\
\hline Control & $120.0(15.2)$ & $114.2(12.8)$ & $110.8(15.0)$ \\
\hline \multicolumn{4}{|c|}{ Diastolic blood pressure [mm Hg]: } \\
\hline Cases & $67.9(7.5)$ & $72.1(10.7)^{\star \&}$ & $74.2(10.0)^{\&}$ \\
\hline Control & $65.4(7.8)$ & $62.8(8.1)^{\star \&}$ & $60.8(11.2)^{\&}$ \\
\hline
\end{tabular}




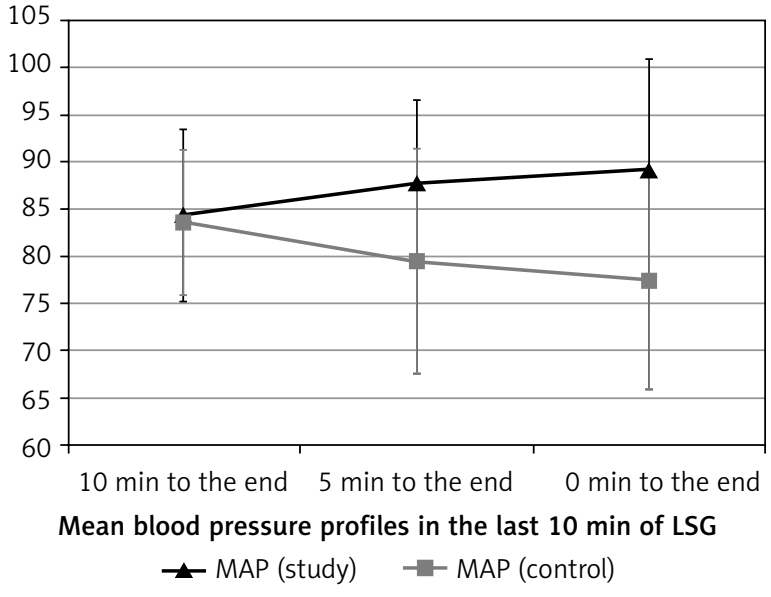

Figure 1. Mean arterial blood pressure measurements of the last 10 min of surgery for patients with $\mathrm{HC}(n=12)$ and their matched controls $(n=12)$

to show increased mean arterial blood pressure in the last 10 min of surgery. The population which reacts to exsufflation by increasing the mean arterial blood pressure is more prone to experience $\mathrm{HC}$ in the postoperative period. This mechanism is not precisely known and needs to be investigated.

Our observation supports widespread practice for bleeding assessment at the end of surgery. Exsufflation of the pneumoperitoneum for 5 to $10 \mathrm{~min}$ leads to all physiologic changes, which may reveal significant bleeding. The main limitations of our study are the retrospective character of the research and the size of the study group. Bleeding complications in our study occurred in $3.0 \%$ of all cases, and it matches the rates in other studies, ranging from $3 \%$ to $5 \%$ [6]. Considering that the database includes data on 867 patients, the amount of cases that qualified for the survey is acceptable.

\section{Conclusions}

Compared with closely matched control subjects, patients with HC after LSG have increased mean arterial pressure 5 min before the end of surgery. This phenomenon has not been reported in the literature before. However, the observed changes were not clinically significant.

\section{Conflict of interest}

The authors declare no conflict of interest.

\section{References}

1. Angrisani L, Santonicola A, lovino P, et al. Bariatric surgery worldwide 2013. Obes Surg 2015; 25: 1822-32.

2. Walędziak M, Różańska-Walędziak A, Kowalewski PK, et al. Present trends in bariatric surgery in Poland. Videosurgery Miniinv 2019; 14: 86-9.

3. Kowalewski PK, Olszewski R, Walędziak MS, et al. Long-term outcomes of laparoscopic sleeve gastrectomy - a single-center, retrospective study. Obes Surg 2018; 28: 130-4.

4. Wojciak PA, Pawłuszewicz P, Diemieszczyk I, et al. Laparoscopic sleeve gastrectomy: a study of efficiency in treatment of metabolic syndrome components, comorbidities and influence on certain biochemical markers. Videosurgery Miniinv 2020; 15: 136-47.

5. Neagoe R, Muresan M, Timofte D, et al. Long-term outcomes of laparoscopic sleeve gastrectomy - a single-centre prospective observational study. Videosurgery Miniinv 2019; 14: 242-8.

6. Shikora SA, Mahoney CB. Clinical benefit of gastric staple line reinforcement (SLR) in gastrointestinal surgery: a meta-analysis. Obes Surg 2015; 25: 1133-41.

7. Janik MMR, Walędziak M, Brągoszewski J, et al. Prediction model for hemorrhagic complications after laparoscopic sleeve gastrectomy: development of SLEEVE BLEED Calculator. Obes Surg 2017; 27: 968-72.

8. Janik MR, Rogula T, Kowalewski P, et al. Case-control study of postoperative blood pressure in patients with hemorrhagic complications after laparoscopic sleeve gastrectomy and matched controls. Obes Surg 2017; 27: 1849-53.

9. Janik $M$, Walędziak $M$, Brągoszewski J, et al. Prediction model for hemorrhagic complications after laparoscopic sleeve gastrectomy: development of SLEEVE BLEED Calculator. Obes Surg 2017; 27: 968-72.

10. Jossart GH. Complications of sleeve gastrectomy: bleeding and prevention. Surg Laparosc Endosc Percutan Tech 2010; 20: 146-7.

11. Kawabata H, Tran M, Hines P, Bristol-Myers S. Using SAS to Match Cases for Case-Control Studies. Pap. 173 -29. Princeton, New Jersey; p. 1-7.

12. Breslow NE, Day NE. International Agency for Research on Cancer. Statistical methods in cancer research Volume I-The Analysis of Case-Control Studies. Stat Methods Cancer Res 1980; 1: 346.

13. Nguyen NT, Wolfe BM. The physiologic effects of pneumoperitoneum in the morbidly obese. Ann Surg 2005; 241: 219-26.

14. Umar A, Mehta KS, Mehta N. Evaluation of hemodynamic changes using different intra-abdominal pressures for laparoscopic cholecystectomy. Indian J Surg 2013; 75: 284-9.

Received: 9.07.2020, accepted: 9.12.2020. 